\title{
$k$-asymmetric spin splitting at the interface between transition metal ferromagnets and heavy metals
}

\author{
Sergiy Grytsyuk, ${ }^{1}$ Abderrezak Belabbes, ${ }^{1}$ Paul M. Haney, ${ }^{2}$ Hyun-Woo Lee, ${ }^{3}$ Kyung-Jin Lee, ${ }^{4,5}$ M. D. Stiles, ${ }^{2}$ \\ Udo Schwingenschlögl, ${ }^{1, *}$ and Aurelien Manchon ${ }^{1, \dagger}$ \\ ${ }^{1}$ King Abdullah University of Science and Technology (KAUST), Physical Science and Engineering Division (PSE), \\ Thuwal 23955-6900, Saudi Arabia \\ ${ }^{2}$ Center for Nanoscale Science and Technology, National Institute of Standards and Technology, Gaithersburg, Maryland 20899-6202, USA \\ ${ }^{3}$ PCTP and Department of Physics, Pohang University of Science and Technology, Kyungbuk 37673, Korea \\ ${ }^{4}$ Department of Materials Science and Engineering, Korea University, Seoul 02841, South Korea \\ ${ }^{5}$ KU-KIST Graduate School of Converging Science and Technology, Korea University, Seoul 02841, Korea
}

(Received 10 December 2015; revised manuscript received 27 April 2016; published 23 May 2016)

\begin{abstract}
We systematically investigate the spin-orbit coupling-induced band splitting originating from inversion symmetry breaking at the interface between a Co monolayer and $4 d(\mathrm{Tc}, \mathrm{Ru}, \mathrm{Rh}, \mathrm{Pd}$, and $\mathrm{Ag}$ ) or $5 d(\mathrm{Re}$, $\mathrm{Os}, \mathrm{Ir}, \mathrm{Pt}$, and $\mathrm{Au}$ ) transition metals. In spite of the complex band structure of these systems, the odd-in- $k$ spin splitting of the bands displays striking similarities with the much simpler Rashba spin-orbit coupling picture. We establish a clear connection between the overall strength of the odd-in- $k$ spin splitting of the bands and the charge transfer between the $d$ orbitals at the interface. Furthermore, we show that the spin splitting of the Fermi surface scales with the induced orbital moment, weighted by the spin-orbit coupling.
\end{abstract}

DOI: 10.1103/PhysRevB.93.174421

\section{INTRODUCTION}

The development of modern spintronic devices, such as magnetic random access memories and current-driven nano-oscillators, currently relies on the exploitation of the mechanism of spin-transfer torque [1] in magnetic systems displaying perpendicular magnetic anisotropy [2]. Besides materials displaying bulk uniaxial magnetic anisotropy (such as ordered alloys [3]), the most promising devices involve multilayers accommodating large interfacial-induced perpendicular magnetic anisotropy, such as superlattices involving two different magnetic transition metals [4], and interfaces between transition-metal ferromagnets and noble metals [5] or metal oxides [6,7] (see also Ref. [8]). This combination of materials in magnetic tunnel junctions has proven successful in reducing the critical current density needed to achieve currentdriven magnetic excitations and switching within a reasonable range (i.e., below $10^{6} \mathrm{~A} / \mathrm{cm}^{2}$ ). However, the difficulty in reducing this critical current density further constitutes a major hurdle for applications, and calls for innovative mechanisms beyond spin-transfer torque. As discussed below, the physics of spin-orbit coupling at the origin of the perpendicular magnetic anisotropy might hold the key to the next technological breakthrough [9].

Interfacial perpendicular magnetic anisotropy at $X / F$ interfaces ( $X$ being a noble metal, and $F$ a transition-metal ferromagnet), defined as the difference $\Delta E$ in the groundstate energy when the magnetization changes from in plane to out of plane, is a subtle phenomenon that arises from spin-orbit coupled orbital overlaps. Spin-orbit coupling, given by $\hat{H}_{\text {so }}=(\xi / \hbar) \hat{\boldsymbol{\sigma}} \cdot(\nabla V \times \hat{\mathbf{p}})(\xi$ is the spin-orbit coupling strength in $\mathrm{eV} / \mathrm{m}^{2}$ ), couples the spin degree of freedom $\sigma$ to the gradient of the crystal field, $\nabla V$. In the spherical atomic potential approximation, $\nabla V=\left(\partial_{r} V / r\right) \hat{\mathbf{r}}$ and the interfacial

\footnotetext{
*udo.schwingenschlog1@kaust.edu.sa

† aurelien.manchon@kaust.edu.sa
}

perpendicular magnetic anisotropy is associated with the orbital overlap leading to an enhanced interfacial orbital angular momentum, $\hat{\mathbf{L}}=\hat{\mathbf{r}} \times \hat{\mathbf{p}}[5,10,11]$. Interfacial perpendicular magnetic anisotropy survives in symmetrically grown systems such as $\mathrm{Pt} / \mathrm{Co} / \mathrm{Pt}$ (e.g., see Refs. [5,12]). However, when the two interfaces embedding the transition-metal ferromagnet are not equivalent, such as in $\mathrm{Pt} / \mathrm{Co} / \mathrm{AlOx}$ or $\mathrm{Ta} / \mathrm{CoFeB} / \mathrm{MgO}$, the symmetry is broken and additional effects emerge.

In such systems, the sharp interface between the ferromagnet and the noble metal breaks the inversion symmetry along the normal to the interface, $\mathbf{z}$. In general, spatial inversion symmetry breaking allows the spin-orbit coupling to develop terms that are odd in momentum $\mathbf{k}$, which is well known in bulk noncentrosymmetric semiconductors [13] and has been recently observed at oxide heterointerfaces [14]. In the case of interfacial symmetry breaking, a simplistic picture suggests that the gradient of potential becomes $\langle\xi \nabla V\rangle \approx-\alpha \mathbf{z}$ and the spinorbit coupling Hamiltonian reduces to $H_{\mathrm{R}} \approx-\alpha \hat{\boldsymbol{\sigma}} \cdot(\mathbf{z} \times \mathbf{k})$, where $\alpha$ is the so-called Rashba parameter $[15,16]$. This coupling results in a $k$ splitting of the band structure, as well as in $k$-dependent spin polarization of the wave functions. Such odd-in- $k$ spin-orbit coupling has been observed at the surface of various metals such as $\mathrm{Au}$ [17], $\mathrm{Gd}$ [18], or $\mathrm{Bi}$ compounds [19,20], and more recently at the surface of three-dimensional topological insulators [21]. It has a dramatic impact on both the equilibrium and nonequilibrium properties of magnetic interfaces.

In equilibrium, this odd-in- $k$ spin-orbit coupling results in the emergence of an antisymmetric magnetic exchange interaction between neighboring spins. This so-called Dzyaloshinskii-Moriya interaction [22] has the form $\sum_{i j} \mathcal{D}_{i j} \mathbf{S}_{i} \times \mathbf{S}_{j}$, where $\mathbf{S}_{i(j)}$ is the direction of the spin angular momentum of the ion at position $\mathbf{r}_{i(j)}$. Under certain conditions (i.e., for certain values of the magnetic exchange, anisotropy, and temperature), this interaction produces chiral spin textures such as spin spirals, as observed at W/Mn and $\mathrm{Ir} / \mathrm{Fe}$ interfaces [23,24], and skyrmions [25]. Very recently, it has also been shown that this interaction favors Néel 
over Bloch configuration of magnetic domain walls in $\mathrm{Ni} / \mathrm{Fe}$ multilayers [26]. Such a distortion is a key element to understand the observed anomalous domain-wall motion in ultrathin perpendicularly magnetized multilayers [27,28].

Out of equilibrium, the interfacial odd-in- $k$ spin-orbit coupling enables the so-called inverse spin galvanic effect, i.e., the electrical generation of a nonequilibrium spin density [29]. This spin density can be used to manipulate the magnetization of the adjacent ferromagnet, a mechanism tagged spin-orbit torque [30]. Such an effect has been theoretically predicted using model Hamiltonians with Rashba or Dresselhaus spinorbit coupling [31,32] and first-principles calculations on realistic interfaces have been reported recently [33], confirming qualitatively the earlier results. Its experimental identification in $\mathrm{Pt} / \mathrm{Co} / \mathrm{AlOx}[34,35]$ and other similar structures [36,37] is currently a growing field in spin electronics and regarded as a possible way to complement or even replace spintransfer torque in devices possessing perpendicular magnetic anisotropy.

While these phenomena all arise from the presence of oddin- $k$ spin-orbit coupling in noncentrosymmetric materials, the details of these mechanisms remain quite complex and explicit connections have only been established within the toy model of Rashba spin-orbit coupling [38]. In fact, first-principles calculations have clearly revealed that the latter approximation is far from realistic [33,39-42]: breaking spatial inversion symmetry hardly affects the strength of the atomic spin-orbit coupling itself (in sharp contrast with the phenomenological picture developed by Bychkov and Rashba [15]), but it modifies the orbital hybridization close to the nucleus, where the spin-orbit coupling is stronger [39-42]. This symmetrybreaking-induced modification of the orbital hybridization results in an effective odd-in- $k$ splitting of the band structure. The magnitude of such splitting scales with the strength of interfacial spin-orbit coupling, which governs the physical phenomena mentioned above.

(a)

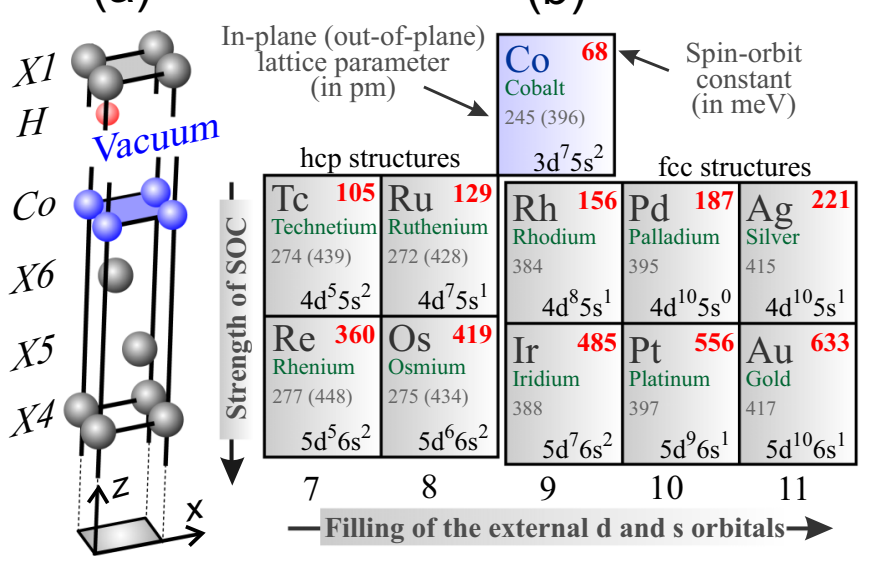

FIG. 1. (a) Unit cell of the $X /$ Co interfaces ( $X=4 d$ metals $=\mathrm{Tc}$, $\mathrm{Ru}, \mathrm{Rh}, \mathrm{Pd}, \mathrm{Ag}$; and $X=5 d$ metals $=\mathrm{Re}, \mathrm{Os}, \mathrm{Ir}, \mathrm{Pt}, \mathrm{Au}$ ) and (b) their electronic configurations used in this work. Numbers in red and gray stand, respectively, for the strength of spin-orbit coupling [44] (in $\mathrm{meV}$ ) and lattice parameters (in pm) obtained in this work. Numbers in brackets are the out-of-plane lattice constants for the hcp structures.
In this paper, we focus on the nature of the band splitting induced by the interfacial symmetry breaking, in order to uncover the physics governing the interfacial spin-orbit coupling in magnetic bilayers. We present a systematic investigation of the (spin-orbit coupling-induced) band splitting at $X / \mathrm{Co}(1 \mathrm{ML})$ interfaces, where $X$ represents a $4 d$ or $5 d$ metal, as depicted in Fig. 1. Using first-principles methods, we establish clear connections between the odd-in- $k$ spin splitting and the interfacial orbital angular momentum and related charge transfer. This paper is organized as follows. Computational details are summarized in Sec. II and the results are reported in Sec. III. Section III A presents the electronic structure of these interfaces together with their magnetization profile, while Sec. III B describes the spin-orbit coupling-induced band-splitting properties. A summary is given in Sec. IV.

\section{COMPUTATIONAL DETAILS}

The accurate description of the magnetic and electronic properties of $X /$ Co interfaces ( $X=4 d$ and $X=5 d$ metals) using first-principles calculations necessitates the definition of a huge supercell in the $(\mathbf{x}, \mathbf{y})$ plane with a Moiré pattern [43] due to the large lattice mismatch between the two materials. However, the large size of the cell combined with the presence of spin-orbit coupling makes such calculations computationally expensive. To avoid this hurdle, we imposed the lattice parameter of the freestanding Co layer to match the lattice parameter of the substrate $X$. Due to the artificial strain, the computed electronic and magnetic properties of the $X /$ Co system may be different from the ones of a realistic interface. Indeed, in Ref. [43], it was demonstrated that for a Co monolayer deposited on top of $\mathrm{Pt}(\mathrm{Au})$, the tensile stress results in an enhancement of the magnetic moments of $\mathrm{Pt}(\mathrm{Au})$ and Co layers by $22 \%(10 \%)$ and $10 \%(15 \%)$, respectively. The objective of the present work is not to match the experimental observations by modeling a realistically disordered interface, but rather to describe the systematic modification of the band splitting and magnetic properties of such bilayers when changing the heavy-metal substrate.

In this work, a monolayer of Co was placed on top of a six-layer slab of an $X(111)$ substrate that is either a $4 d(\mathrm{Tc}, \mathrm{Ru}, \mathrm{Rh}, \mathrm{Pd}$, and $\mathrm{Ag}$ ) or a $5 d(\mathrm{Re}, \mathrm{Os}, \mathrm{Ir}, \mathrm{Pt}$, and $\mathrm{Au}$ ) metal; see Fig. 1(a). This allows us to study the dependence of the band splitting and its related properties as a function of the spin-orbit coupling strength and electronic configurations of the external $d s$ orbitals, as illustrated in Fig. 1(b). Moving from the left to the right side of the table, the number of electrons on the external $d s$ orbitals grows from 7 to 11 . Structures with $[d s]^{7}$ and $[d s]^{8}$ electronic configurations have hcp packing, while those with $[d s]^{9}$, $[d s]^{10}$, and $[d s]^{11}$ electronic configurations adopt fcc packing. Our test calculations reveal that Co prefers hcp and fcc hollow sites on top of $X$ in the first and second cases, respectively. In addition, a vacuum of $10 \AA$ and one $H$ atom on the bottom of the substrate were found as sufficient conditions to avoid charge accumulation. First-principles calculations were performed in the generalized gradient approximation [45]. For structure optimization and computations of magnetic properties, we used the pseudopotential method implemented 
in VASP [46-48]. In addition, for the sake of comparison, the magnetic properties were also investigated within the full-potential code FLEUR [49,59]. The spin-orbit coupling was treated using the second variational approach. We verified that dipole corrections do not affect our results and we expect the influence of orbital polarization to be insignificant due to the small orbital moment in our system.

Local spin $(S)$ and orbital $(L)$ moments are obtained by integrating the corresponding densities in a sphere centered around each atom: $S=\mu_{B} \int\left[n^{\uparrow}(r)-n^{\downarrow}(r)\right] d r$ and $L=$ $\int \ell(r) d r$. Here, $n(r)^{\uparrow(\downarrow)}$ are spin-up (-down) densities and $\ell=-\mu_{B} \sum_{i}\left\langle\psi_{i}|\hat{L}| \psi_{i}\right\rangle$ is orbital magnetization density, with $\left|\psi_{i}\right\rangle$ being an eigenstate of the system and $\hat{L}$ the angular orbital momentum operator. The summations run over the occupied states and include all orbitals.

The magnetic properties were calculated using an increased number of $k$ points in the Brillouin zone until convergence, which is achieved for $2304 k$ points. To study the band splitting below and at Fermi level, we used $256 k$ points for each radial direction and 4096 equally distributed $k$ points in the irreducible Brillouin zone, respectively.

\section{RESULTS AND DISCUSSION}

\section{A. Electronic structures}

The magnetic properties of $X / \mathrm{Co}$ interfaces are direct outcomes of interfacial orbital overlap. Therefore, before entering into the detailed analysis of these properties, we turn our attention towards the nature of these hybridizations. The density of states and magnetic properties of the $d$ orbitals of $X /$ Co bilayers are displayed in Figs. 2 and 3, respectively. The first remarkable feature is the distinct behavior between metals with partially filled $d$ shells (Tc, Ru, Re, Os, Ir, Pt, Rh, and $\mathrm{Pd}$ ) and metals with filled $d$ shells ( $\mathrm{Au}$ and $\mathrm{Ag}$ ). While the former present a sizable density of states at Fermi energy, this density of states is vanishingly small in the latter cases, leading to a reduced $d$-orbital hybridization between Co and $X$. As a general rule, upon increasing the $d$-orbital filling, the binding energy decreases due to (i) the enhancement of the "artificial" lattice distortion imposed by the lattice mismatch between Co and $X$, and (ii) the reduction of the orbital hybridization. The latter results in a strong reduction of the $\mathrm{Co}-X$ binding in the case of $\mathrm{Au}$ and $\mathrm{Ag}$.

The nature of the orbital hybridization between Co and $X$ has important consequences for the magnetic properties at the interface. First, metals with partially filled $d$ shells acquire a large magnetic moment through the proximity effect [10,50-52], which vanishes in the case of metals with filled $d$ shells (Au and Ag); see Fig. 3(a). Notice that the magnetic proximity effect is very small in the case of Tc, Ru, Os, and Re, but does not vanish. Most importantly for the present study, the magnetic proximity effect is accompanied by an induced orbital moment that is negative for Tc, Ru, Re, and $\mathrm{Os}$, and positive at the interface with $\mathrm{Rh}, \mathrm{Pd}, \mathrm{Ag}, \mathrm{Ir}, \mathrm{Pt}$, and $\mathrm{Au}$; see Fig. 3(b). As discussed below, the sign and magnitude of the induced orbital moment have a dramatic impact on the spin-orbit-induced splitting of the band structure.

It is worth mentioning that all of the structures discussed in this work and involving heavy metals with partially filled $d$
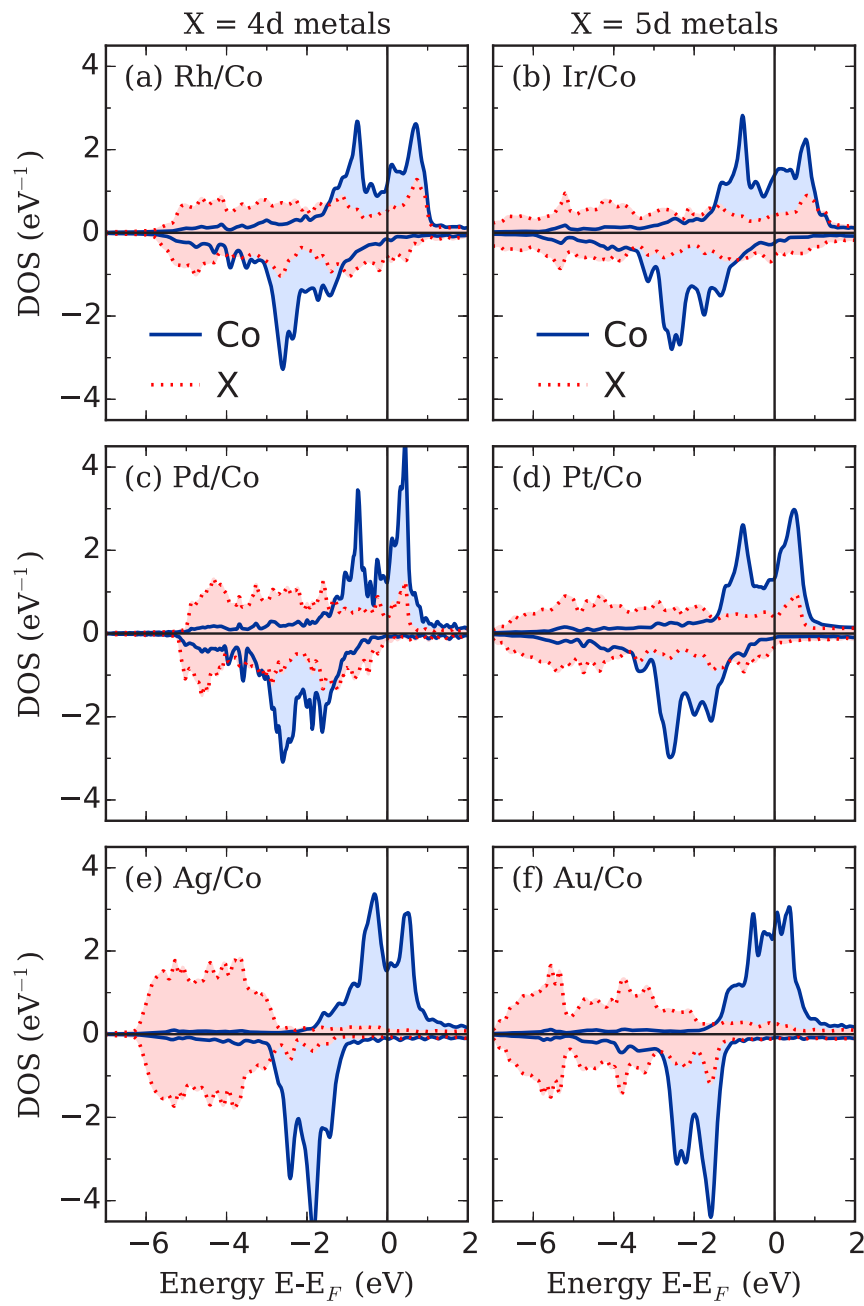

FIG. 2. Projected density of states (DOS) for the interfacial $X$ and Co layers at the $X /$ Co interfaces, where $X$ is a heavy metal, as indicated in the figure. The amount of hybridization between the $3 d$ orbitals of Co and 4(5) $d$ orbitals of the heavy metal decreases when increasing the $d$-orbital filling of the heavy metal. The densities of states for $X=\mathrm{Tc}, \mathrm{Ru}, \mathrm{Re}$, and Os are not represented as they show a qualitatively similar behavior as heavy metals with partially filled $d$ shells.

shells present interfacial perpendicular magnetic anisotropy, while the weak hybridization between Co and metal with filled $d$ orbitals ( $\mathrm{Ag}$ and $\mathrm{Au}$ ) results in large in-plane magnetic anisotropy (the latter being in contradiction with experimental observations, but in agreement with other theoretical results due to artificial strain in our calculations [52-54]). However, we could not find any robust correlation between the magnetic anisotropy and the strength of the band splitting.

\section{B. $k$-asymmetric spin splitting}

In the previous section, we have illustrated the complex hybridization scheme of heavy-metal/ferromagnet interfaces through the onset of the magnetic proximity effect as well as induced orbital moment. We now turn our attention towards the main topic of the present work, i.e., the nature of spinorbit coupling-induced band splitting in asymmetric magnetic bilayers. 

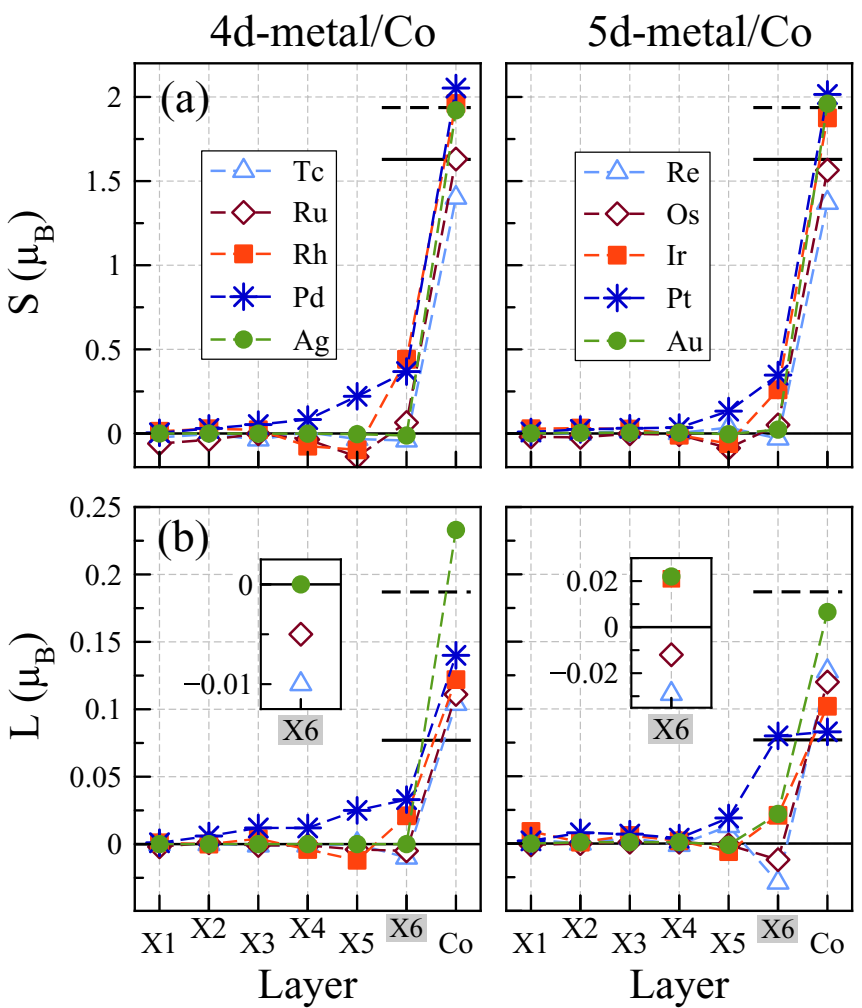

FIG. 3. Layer-resolved magnetic properties of the $X /$ Co interfaces, where $X$ is a heavy metal. (a) Spin and (b) orbital contributions to the magnetic moment for a magnetization pointing along $\mathbf{x}$. The left (right) panels represent the $4 d(5 d)$ metals, as indicated in the figure. The solid (dashed) line indicates the value for the bulk (freestanding layer of) Co.

\section{Spin-orbit-induced band splitting}

In inversion asymmetric systems, spin-orbit coupling induces a spin splitting of the band structure of the form $H_{\mathrm{so}}=\mathbf{w}(\mathbf{k}) \cdot \boldsymbol{\sigma}$, where $\mathbf{w}(\mathbf{k})=-\mathbf{w}(-\mathbf{k})$ is an odd function of k. In order to visualize and analyze such a band splitting, we adopt the approach developed in Ref. [55]: The band structure is calculated for two opposite directions of the magnetization, say $\pm \mathbf{u}$, and hence reveals the spin-orbit coupling-induced asymmetric band splitting. For instance, the Fermi surface and band structures of the Ir/Co interface along the $x$ and $y$ directions in the Brillouin zone are reported in Fig. 4 (central, top, and right panels, respectively) when imposing the magnetization to lie along $+\mathbf{x}$ (red lines) and $-\mathbf{x}$ (blue lines). The band structures obtained for opposite magnetization directions are mirror symmetric with respect to $k_{y}=0$, as expected, ensuring that the band structure remains timereversal symmetric, i.e., $\delta E\left(m_{x}, k_{y}\right)=\delta E\left(-m_{x},-k_{y}\right)$. When the magnetization is applied along $\pm \mathbf{x}$, the band structure calculated along the $x$ direction in the Brillouin zone does not display any distortion; see Fig. 4, top panel. Similar results have been obtained for all the substrates considered. When removing the interface, using either an isolated Co or $X$ layer, this distortion disappears (not shown). Note that in principle, a spin-orbit splitting is also present at the bottom interface. Nevertheless, since band distortion only occurs upon both broken inversion symmetry and broken time-reversal
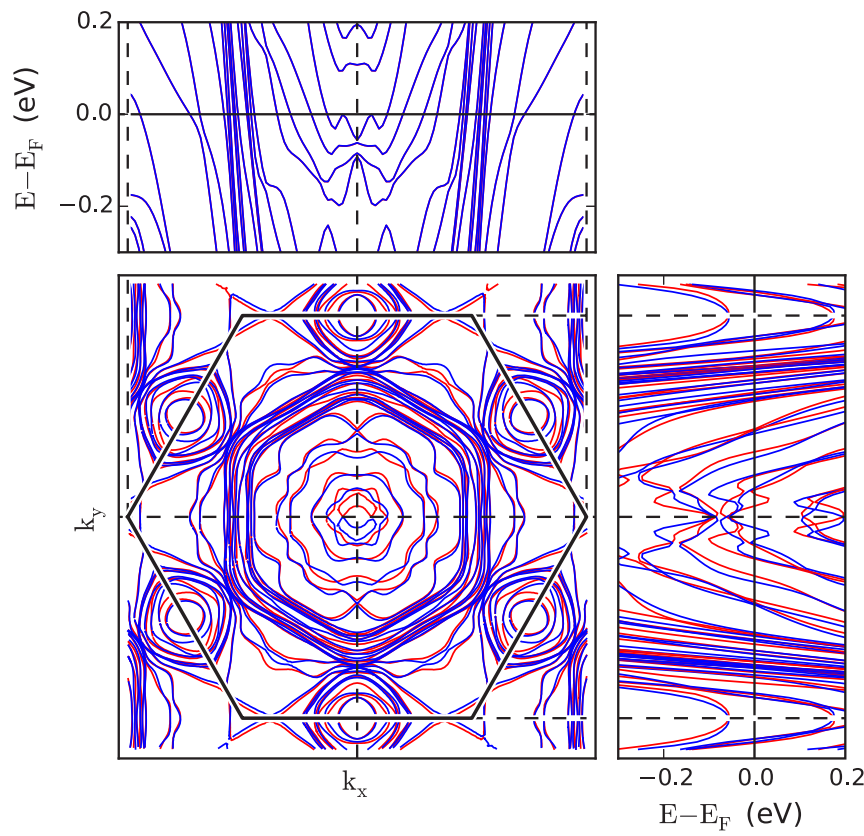

FIG. 4. Central panel: two-dimensional Fermi surface of Ir/Co interface in $\left(k_{x}, k_{y}\right)$ plane, when the magnetization direction is along $+\mathbf{x}$ (red lines) or $-\mathbf{x}$ (blue lines). Right panel: Band structure of Ir/Co interfaces calculated along the $y$ direction in the Brillouin zone. Top panel: Band structure of Ir/Co interfaces calculated along the $x$ direction in the Brillouin zone. A clear distortion of the band structure is observed when projected perpendicular to the magnetization direction (right panel), while no such modification appears when the band structure is projected along the magnetization direction (top panel)

invariance, the spin-orbit splitting at the bottom interface does not create a band distortion.

A direct evaluation of the odd-in- $k$ band splitting from first principles is only achievable in systems presenting a free-electron-like dispersion [18,56]. For systems displaying more complex band structures, alternative methods have been considered. For instance, Bihlmayer et al. [39] directly calculated the potential gradient $\left\langle\partial_{r} V / r\right\rangle$ close to the atom nucleus, Park et al. [55] evaluated the linear slope of the band structure close to $k=0$ (which probably corresponds to the closest definition of the Rashba spin splitting), while Haney et al. [33] and Tokatly et al. [42] calculated the inverse spin galvanic effect arising from odd-in- $k$ spin splitting (which is a more experimentally relevant quantity). The variety of methods illustrates the difficulty to give a proper account of the strength of spin-orbit coupling-induced spin splitting in asymmetric systems. In the present work, we adopt an intuitive approach to evaluate the magnitude of the spin splitting at and below Fermi level.

\section{Spin splitting below Fermi level}

The area spanned by the band $n$ upon magnetization reversal from $+\mathbf{u}$ to $-\mathbf{u}$ is $\mathcal{A}_{n}(\beta)=\int\left|E_{n}^{+} f_{n}^{+}-E_{n}^{-} f_{n}^{-}\right| d k_{\beta}$ [shaded area in Fig. 5(a)], where $E_{n}^{ \pm}$is the energy dispersion when the magnetization lies along $\pm \mathbf{u}, f_{n}^{ \pm}=1$ for states below Fermi level, and 0 otherwise. The $k$ integration is performed along a direction of the two-dimensional Brillouin zone defined by 


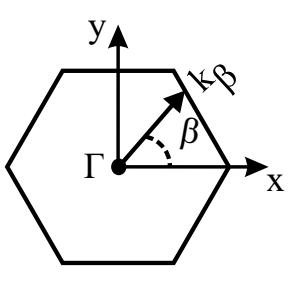

(a)

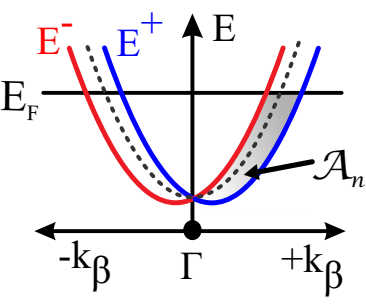

(c) $\mathrm{m} \| \mathrm{x}$

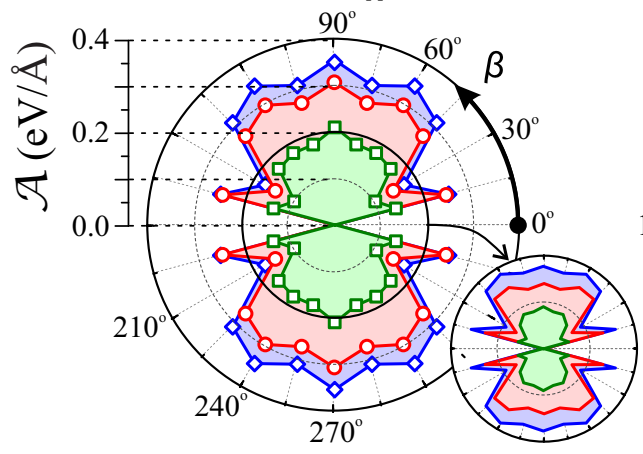

(d) $\mathrm{m} \mathrm{\|} \mathrm{y}$

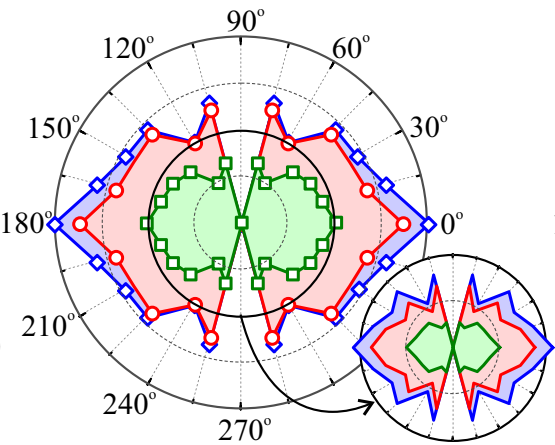

(b)

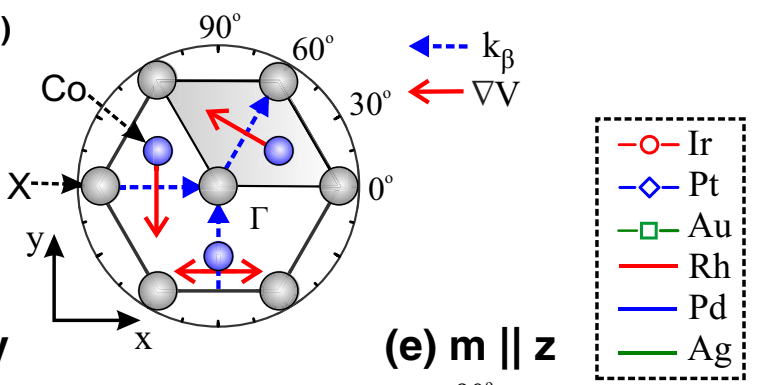

FIG. 5. (a) Brillouin zone and schematic representation of the odd-in- $k$ splitting along the $\beta$ direction. $\mathcal{A}_{n}$ is the spanned area. (b) $(x, y)$ projection of the $X /$ Co interface. $k_{\beta}$ are examples of the electron propagation directions with respect to the crystal structure. (c)-(e) $\mathcal{A}=\mathcal{A}(\beta)$, for the magnetization along the $+\mathbf{x},+\mathbf{y}$, and $+\mathbf{z}$ directions, respectively. Small and large polar coordinates have the same scale and refer to the $4 d$-metal/Co and $5 d$-metal/Co interfaces, respectively. For the sake of readability, the values of $\mathcal{A}$ for Tc, Ru, Os, and Re are omitted in this figure.

the angle $\beta$ [see Fig. 5(a)]. To evaluate the global strength of the spin splitting below the Fermi level, we calculate the total area $\mathcal{A}(\beta)$ as

$$
\mathcal{A}(\beta)=\sum_{n}^{N} \sum_{i}^{N_{k}}\left|E_{n i}^{+} f_{n i}^{+}-E_{n i}^{-} f_{n i}^{-}\right| \Delta k_{i},
$$

where the first summation $\sum_{n}^{N}$ runs over the band index $n$, and the second summation $\sum_{i}^{N_{k}}$ stems from the discretization of the integral in $k$ space along the direction $\beta$. Notice that in Eq. (1), only absolute values of the energy shifts enter the calculation. Indeed, since the sign of the spin splitting depends on the band index [55], accounting for the relative magnitudes of the shifts rather than for their absolute values would result in cancellations between different bands and might not give a full account of the global spin-splitting strength. Therefore, $\mathcal{A}(\beta)$ provides an estimation of the spin-splitting asymmetry for all bands below the Fermi energy.

In contrast to systems that have a well-isolated surface state (such as Au (111) surface [17] or Bi/Ag (111) surface alloy [19]), in the case of $X /$ Co bilayers, the strong interfacial spin-orbit splitting is spread over all of the states of the slab (see also Ref. [42]). While the spin-orbit coupling affects states near the interface, all of the states are delocalized over the whole slab, so the splitting in individual states shrinks as the number of layers in the slab increases. However, the total effect of the interface spin-orbit coupling, $\mathcal{A}(\beta)$, converges quickly as the thickness of the substrate increases [shown in Figs. 6(a) and 6(b)] because the decrease in the contribution per state is compensated by the increase in the number of states.

To numerically compute $\mathcal{A}(\beta)$, the band structure calculations were repeated for different directions $\beta$ in the Brillouin zone [see Fig. 5(a)], as well as for three different magnetization directions (along the $\mathbf{x}, \mathbf{y}$, and $\mathbf{z}$ axes). The spanned absolute areas $\mathcal{A}(\beta)$ calculated for various $\beta$ directions in the Brillouin
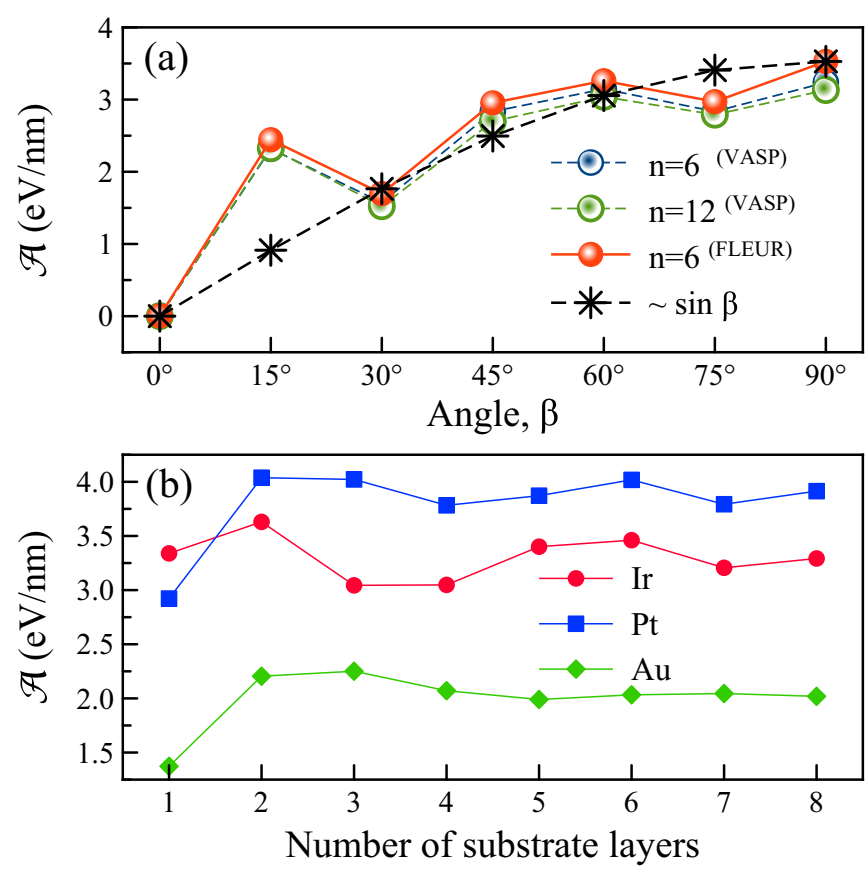

FIG. 6. (a) Angular dependence of the total band splitting $\mathcal{A}(\beta)$ calculated by first principles (VASP and FLEUR) and by the standard Rashba model ( $H_{\mathrm{R}} \propto \sin \beta$; see main text) for the $\mathrm{Pt}_{n} /$ Co interfaces ( $n$ is a number of Pt layers). Magnetization is along $\mathbf{x}$. (b) Dependence of $\mathcal{A}\left(\beta=0^{\circ}\right)$ on the number of substrate layers for Ir, Pt, and $\mathrm{Au}$. Magnetization is along $\mathbf{y}$. 
zone and when the magnetization lies along the $\mathbf{x}, \mathbf{y}$, and $\mathbf{z}$ axes are shown in Figs. 5(c)-5(e), respectively. Several features are worth noticing.

First, while the global angular dependence of $\mathcal{A}(\beta)$ depends on the magnetization direction and displays different symmetries in the different cases, their shapes do not depend on the substrate. Second, when the magnetization lies in the interface plane $(\mathbf{x}, \mathbf{y}), \mathcal{A}(\beta)$ reaches its maximum when $\mathbf{m} \perp \mathbf{k}\left(\beta= \pm 90^{\circ}\right.$ when $\mathbf{m} \| \mathbf{x}$ and $\beta=0^{\circ}$ when $\left.\mathbf{m} \| \mathbf{y}\right)$ and vanishes when $\mathbf{m} \| \mathbf{k}$ $\left(\beta=0^{\circ}\right.$ when $\mathbf{m} \| \mathbf{x}$ and $\beta= \pm 90^{\circ}$ when $\left.\mathbf{m} \| \mathbf{y}\right)$. These two features partially validate a standard Rashba model described by the Hamiltonian $H_{\mathrm{R}}=\alpha(\sigma \times \mathbf{k}) \cdot \mathbf{z} \propto \sin \beta$. Figure 6(a) compares the angular dependence of the magnitude of $\mathcal{A}(\beta)$ extracted from the first-principles calculations (from both VASP and FLEUR codes) reported in Fig. 5(c) and the $\sin \beta$ dependence expected from the Rashba model. Deviations from the sine shape are clearly visible in Fig. 6(a) at $\beta=15^{\circ}, 45^{\circ}$, and $75^{\circ}$. These angles correspond to points where the in-plane crystalline symmetry is broken; see Fig. 5(b). Notice that these calculations have been reproduced for a thicker substrate thickness [ $n=12$ layers; green symbols in Fig. 6(a)] with only minor variations, showing that $\mathcal{A}(\beta)$ is a robust quantity to characterize the total band splitting below the Fermi level. Third, a spin splitting is observed when the magnetization lies out of the plane of the interface [see Fig. 5(e)]. In fact, the spin splitting in this case displays a sixfold symmetry, which is a reminiscence of the crystal structure [see Fig. 5(c)]. The vanishing splitting at $\beta=30^{\circ}+m \times 60^{\circ}, m \in \mathbb{Z}$, corresponds to high-symmetry points in the Brillouin zone, while the maxima at $\beta=m \times 60^{\circ}$ corresponds to low-symmetry points [see Fig. 5(b)]. Of course, in a system with cylindrical symmetry around $\mathbf{z}$ such as a two-dimensional free-electron gas with Rashba spin-orbit coupling, no splitting is observed when the magnetization lies along $\mathbf{z}$.

Finally, one can notice that the magnitude of $\mathcal{A}(\beta)$ also depends strongly on the substrate, as reported in Fig. 7(a). Generally, $4 d$ substrates display weaker spin splitting than $5 d$ substrates, which is a direct consequence of their weaker spin-orbit coupling strength. However, the spin-splitting magnitude also depends on the band filling and within a same class of materials (either $4 d$ or $5 d$ ) shows a maximum for $n[d s]^{10}$ materials (i.e., $\mathrm{Pd}$ and $\mathrm{Pt}$ ); see Fig. 1. As mentioned in Sec. I, Bihlmayer et al. [39] and, more recently, Krasovskii [42,57] have noticed that in contrast to the conventional phenomenology of Rashba spin-orbit coupling, the interfacial symmetry breaking leaves the atomic spin-orbit coupling unaffected while it strongly affects the wave function itself by modifying the orbital hybridization. The interplay between this modified wave function and the spherically symmetric spin-orbit coupling produces the asymmetric spin splitting of the band structure. The orbital hybridization at the interface between the substrate and the Co monolayer is associated with a charge transfer from the substrate to the Co layer, which is reported in Fig. 7(a) for $d$ orbitals. A qualitative correlation is obtained between the spin-splitting parameter $\mathcal{A}\left(\beta=90^{\circ}\right)$ and the charge transfer, both displaying a maximum for $\mathrm{Pd}$ and $\mathrm{Pt}$.

\section{Spin splitting at Fermi level}

Let us now turn our attention towards the properties of the Fermi surface, displayed in the central panel of Fig. 4, for Ir/Co.

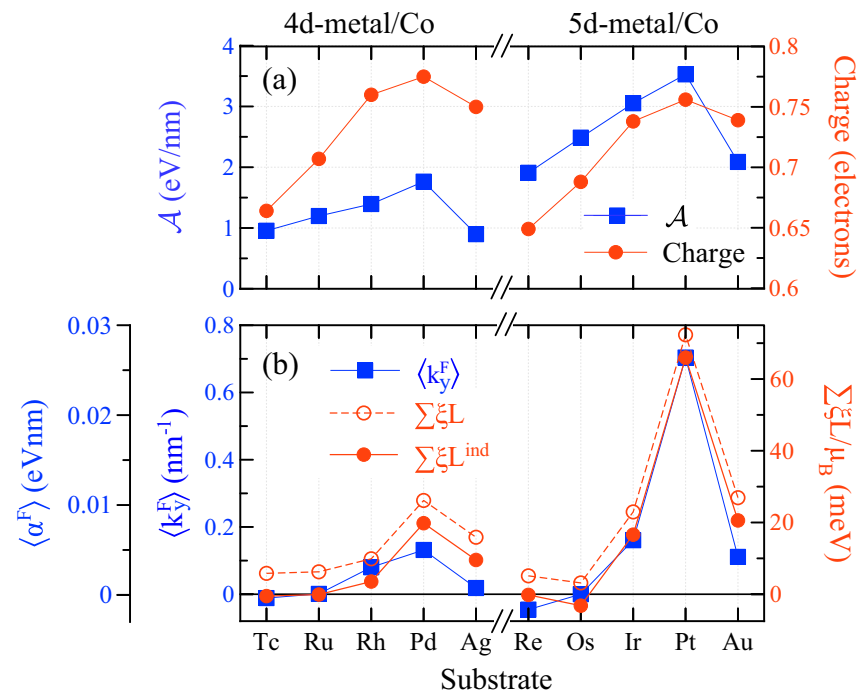

FIG. 7. Correlation between odd-in- $k$ spin splitting and interfacial electronics properties as a function of substrate. (a) Spanned area $\mathcal{A}\left(\beta=90^{\circ}\right)$ (blue symbols) and charge transfer from the $d$ orbitals of substrate to the $d$ orbitals of Co (red symbols). (b) Effective momentum shift $\left\langle k_{y}^{\mathrm{F}}\right\rangle$ (blue symbols) and summation of the induced orbital moments of each atom weighted by their spin-orbit coupling constant, $\sum_{i} \xi_{i} L_{i}^{\text {ind }}$ (red symbols).

While the Fermi surface is very far from circular, it displays a shift along the $y$ direction when changing the magnetization direction from $+\mathbf{x}$ (red) to $-\mathbf{x}$ (blue), confirming the intuition given by the Rashba model. To evaluate the strength of the spin splitting at the Fermi level, we compute the $k$-averaged Fermi wave vector $\left\langle\mathbf{k}^{\mathrm{F}}\right\rangle$ defined as

$$
\left\langle\mathbf{k}^{\mathrm{F}}\right\rangle=\sum_{n}^{N_{b}} \mathbf{k}_{n}^{\mathrm{F}}=\sum_{n}^{N_{b}} \frac{1}{N_{k}} \sum_{i}^{N_{k}} \mathbf{k}_{i n}^{\mathrm{F}},
$$

where the first summation $\sum_{n}^{N_{b}}$ runs over the band index $n$ and the second one $\sum_{i}^{N_{k}}$ stems from the discretization of the integral in $k$ space. This parameter quantifies the center of mass of the Fermi surface. If there is no spin splitting, $\left\langle\mathbf{k}^{\mathrm{F}}\right\rangle=0$. In the present case, the magnetization is along $\pm \mathbf{x}$ so that $\left\langle\mathbf{k}^{\mathrm{F}}\right\rangle=\left\langle k_{y}^{\mathrm{F}}\right\rangle \mathbf{y}$, as in Fig. 4. The calculated $\left\langle k_{y}^{\mathrm{F}}\right\rangle$ is reported in Fig. 7(b) for different substrates. While it is quite small in the case of $4 d$ metals, it is much larger for $5 d$ metals. In both cases, the maximum is reached for $[d s]^{10}$ configurations, corresponding to Pd and Pt substrates.

Since the electronic states at the Fermi level can change their occupancy more easily, they are more likely to participate in charge transfer and therefore to be affected by the interfacial potential gradient $\nabla_{z} V$. The charge transfer scales with the strength of the orbital hybridization and results in an enhancement of the orbital angular momentum on the substrate, as reported in Fig. 3(b). Park et al. [58] proposed that at a surface, the local orbital momentum in the presence of a surface-normal electric field (due to inversion symmetry breaking) results in Rashba-type splitting. Following this idea, we assume that the strength of the band splitting for the $X / \mathrm{Co}$ bilayers should be proportional to $\sum_{i} \xi_{i} L_{i}$, where $L_{i}$ and $\xi_{i}$ are the atomic orbital moment [Fig. 3(b)] and spin-orbit coupling 
constant [Fig. 1(b)], respectively, in each layer $i$. The quantity $\sum_{i} \xi_{i} L_{i}$ for the $X /$ Co interfaces is displayed in Fig. 7(b) (open red symbols) and shows good correlation with $\left\langle k_{y}^{\mathrm{F}}\right\rangle$. However, $\sum_{i} \xi_{i} L_{i}$ is shifted with respect to $\left\langle k_{y}^{\mathrm{F}}\right\rangle$ and is always positive, while $\left\langle k_{y}^{\mathrm{F}}\right\rangle$ reaches negative values for some of the $X /$ Co interfaces. We find that this shift can be eliminated by replacing the total orbital moment $L_{i}$ by the induced orbital moment, $L_{i}^{\text {ind }}=L_{i}-L_{i}^{\text {bulk }}$, where $L_{i}^{\text {bulk }}$ is the value of the orbital momentum in the bulk of layer $i$ (either bulk $X$ or bulk Co). Quantity $\sum_{i} \xi_{i} L_{i}^{\text {ind }}$ is displayed in Fig. 7(b) (filled red symbols) and shows an excellent correlation with $\left\langle k_{y}^{\mathrm{F}}\right\rangle$, i.e., $\left\langle k_{y}^{\mathrm{F}}\right\rangle \approx \eta \sum_{i} \xi_{i} L_{i}^{\text {ind }}$, where $\eta \mu_{B} \approx 10.7(\mathrm{eV} \mathrm{nm})^{-1}$ (calculated in this work). Thus, the presence of the induced orbital moment is a necessary condition for the onset of $k$-asymmetric band splitting.

In order to complete our description of the physics involved, we present the layer contribution of $\xi_{i} L_{i}^{\text {ind }}$ in Fig. 8. First we note that the substrate contribution to the band splitting is large in the case of $5 d$ metals, while in the case of $4 d$ metals, it is comparable with a Co contribution. For instance, at the $\mathrm{Ag} / \mathrm{Co}$ interface, $\mathrm{Ag}$ is not magnetized $\left(L_{\mathrm{Ag}}^{\mathrm{ind}}=0\right)$, and thus the main contribution comes from $\mathrm{Co}$. At the $\mathrm{Pd} / \mathrm{Co}$ interface, in contrast, the induced orbital momentum of $\mathrm{Pd}$ does not vanish away from the interface [see Fig. 3(b)]. Thus the Pd/Co interface has the largest strength of the band splitting among other $4 d$-metal/Co interfaces [see Fig. 7(b)]. Regarding $5 d$ metal/Co interfaces, the largest $\xi_{i} L_{i}^{\text {ind }}$ is observed at the Pt/Co interface, associated with a large induced orbital momentum [see Fig. 3(b)]. Moreover, one can see from Fig. 8 that the induced orbital momentum of $[d s]^{7-8}$ substrates (Tc, Ru, Re, and Os) is negative, while it is positive in the case of $[d s]^{8-10}$ substrates ( $\mathrm{Rh}, \mathrm{Pd}, \mathrm{Ag}, \mathrm{Ir}, \mathrm{Pt}$, and $\mathrm{Au}$ ). This nicely correlates

(a) 4d-metal/Co

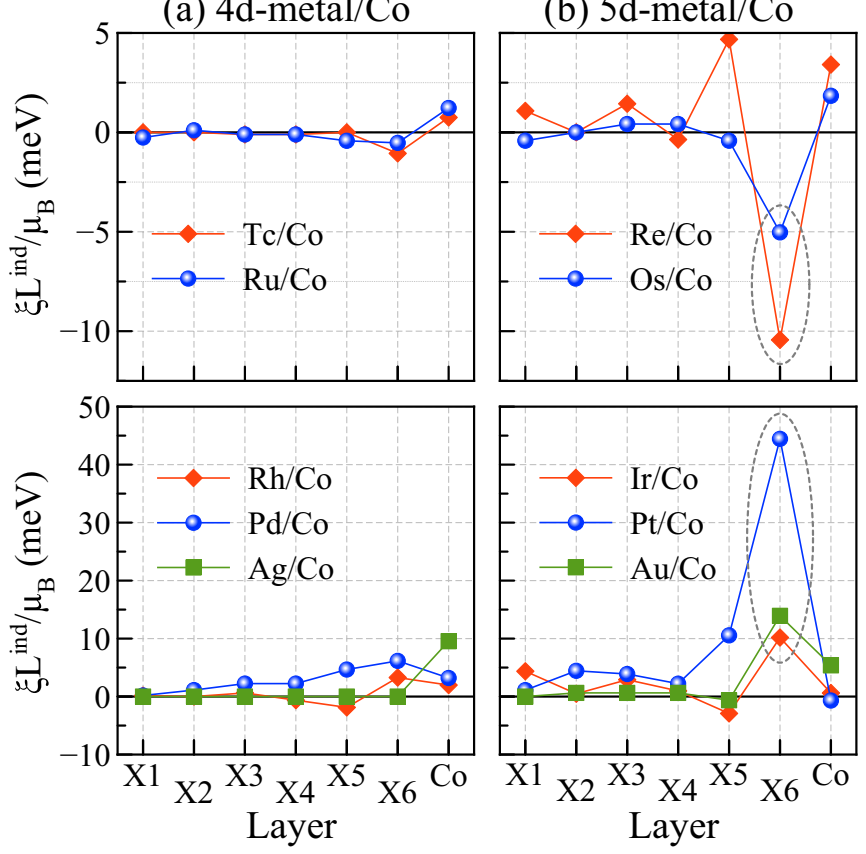

FIG. 8. Induced orbital moment weighted by spin-orbit coupling for (a) $5 d$-metal/Co and (b) $4 d$-metal/Co interfaces. Induced orbital moment for each $i$ is $L_{i}^{\text {ind }}=L_{i}^{X / \text { Co }}-L_{i}^{\text {bulk }}$. with the sign of $\left\langle k_{y}^{\mathrm{F}}\right\rangle$ for the $X / \mathrm{Co}$ interfaces reported in Fig. 7(b).

We conclude this study by evaluating the effective Rashba parameter resulting from the $k$-asymmetric band splitting. Although the band structure of $X /$ Co bilayers is much more complex than the free-electron model, the Rashba parameter is commonly used in experiments to quantify nonequilibrium properties related to interfacial spin-orbit coupling such as the inverse spin galvanic effect. The Rashba parameter at Fermi energy $\left\langle\alpha^{\mathrm{F}}\right\rangle$ can be connected with the parameter $\left\langle k^{\mathrm{F}}\right\rangle$ defined in Eq. (2),

$$
\left\langle\alpha^{\mathrm{F}}\right\rangle=\frac{\hbar^{2}}{2} \sum_{n}^{N_{b}} \frac{1}{N_{k}} \sum_{i}^{N_{k}} \frac{k_{n i}^{\mathrm{F}}}{m_{n i}^{*}} \approx \frac{\hbar^{2}}{2 m_{e}}\left\langle k^{\mathrm{F}}\right\rangle .
$$

The result is shown in Fig. 7(b), assuming the same free-electron effective mass $m^{*}=m_{e}$ for all systems. The largest Rashba parameter is obtained for $\mathrm{Pt} / \mathrm{Co}\left(\left\langle\alpha^{\mathrm{F}}\right\rangle_{\mathrm{Pt}} \approx\right.$ $\left.25 \times 10^{-3} \mathrm{eV} \mathrm{nm}\right)$, while $\mathrm{Ir} / \mathrm{Co}, \mathrm{Au} / \mathrm{Co}$, and $\mathrm{Pd} / \mathrm{Co}$ have a much smaller parameter $\left(\approx 5 \times 10^{-3} \mathrm{eV} \mathrm{nm}\right)$. These values are smaller than the one reported in Ref. [55] for $\mathrm{Pt} / \mathrm{Co}$ $\left(\approx 100 \times 10^{-3} \mathrm{eV} \mathrm{nm}\right)$. These estimations must be handled with sane skepticism (in realistic metals, $m^{*} \ll m_{e}$, and lattice distortions are disregarded in our calculations), but can be compared with the effective parameters extracted experimentally from current-driven field measurements, i.e., $100 \times 10^{-3} \mathrm{eV} \mathrm{nm}$ for Pt/Co [34] and $36 \times 10^{-3} \mathrm{eV} \mathrm{nm}$ for $\mathrm{Pd} / \mathrm{Co} / \mathrm{Pd}$ asymmetric interfaces [36].

\section{DISCUSSION AND CONCLUSION}

In this work, we systematically studied the band splitting originating from spatial inversion symmetry breaking in the presence of spin-orbit coupling in transition-metal bilayers involving $4 d$ ( $\mathrm{Tc}, \mathrm{Ru}, \mathrm{Rh}, \mathrm{Pd}$, and $\mathrm{Ag}$ ) and $5 d$ ( $\mathrm{Re}, \mathrm{Os}$, $\mathrm{Ir}, \mathrm{Pt}$, and $\mathrm{Au}$ ) heavy metals, capped by a monolayer of Co. Our results emphasize the limits of the Rashba scenario at crystalline interfaces involving strong interfacial orbital overlaps. Indeed, the $k$-asymmetric spin splitting is, in general, not linear in $k$ and depends on the band index, and spin splitting is also obtained when the magnetization lies perpendicular to the interface, due to in-plane crystalline symmetry breaking [Fig. 5(d)]. Notwithstanding the crystalline symmetries, the overall angular variation of the $k$-asymmetric spin splitting displays remarkable similarities with what is expected from the much simpler Rashba model (Fig. 6). Finally, our study reveals the crucial role of interfacial orbital overlap in the onset of $k$-asymmetric spin splitting and a phenomenological correlation between the splitting strength and the spin-orbit coupling-weighted induced orbital momentum as well as $d$-orbital charge transfer (Fig. 7).

\section{ACKNOWLEDGMENTS}

S.G. and U.S. acknowledge support from the King Abdullah University of Science and Technology (KAUST). A.B. and A.M. were supported by King Abdullah University of Science and Technology (KAUST) through Award No. OSR-CRG URF/1/2285-01 from the Office of Sponsored Research (OSR). H.-W.L. was supported by the National Research 
Foundation of Korea (NRF) (Grants No. 2011-0030046 and No. 2013R1A2A2A05006237). K.-J.L. was supported by the
National Research Foundation of Korea (NRF) (Grant No. 2015M3D1A1070465).
[1] J. C. Slonczewski, J. Magn. Magn. Mater. 159, L1 (1996); L. Berger, Phys. Rev. B 54, 9353 (1996).

[2] H. Yoda, T. Kishi, T. Nagase, M. Yoshikawa, K. Nishiyama, E. Kitagawa, T. Daibou, M. Amano, N. Shimomura, S. Takahashi, T. Kai, M. Nakayama, H. Aikawa, S. Ikegawa, M. Nagamine, J. Ozeki, S. Mizukami, M. Oogane, Y. Ando, S. Yuasa, K. Yakushiji, H. Kubota, Y. Suzuki, Y. Nakatani, T. Miyazaki, and K. Ando, Curr. Appl. Phys. 10, e87 (2010).

[3] T. Seki, S. Mitani, K. Yakushiji, and K. Takanashi, Appl. Phys. Lett. 88, 172504 (2006).

[4] F. Gimbert and L. Calmels, Phys. Rev. B 86, 184407 (2012); S. Girod, M. Gottwald, S. Andrieu, S. Mangin, J. McCord, Eric E. Fullerton, J.-M. L. Beaujour, B. J. Krishnatreya, and A. D. Kent, Appl. Phys. Lett. 94, 262504 (2009).

[5] D. Weller, Y. Wu, J. Stöhr, M. G. Samant, B. D. Hermsmeier, and C. Chappert, Phys. Rev. B 49, 12888 (1994).

[6] S. Monso, B. Rodmacq, S. Auffret, G. Casali, F. Fettar, B. Gilles, B. Dieny, and P. Boyer, Appl. Phys. Lett. 80, 4157 (2002); B. Rodmacq, A. Manchon, C. Ducruet, S. Auffret, and B. Dieny, Phys. Rev. B 79, 024423 (2009).

[7] S. Ikeda, K. Miura, H. Yamamoto, K. Mizunuma, H. D. Gan, M. Endo, S. Kanai, J. Hayakawa, F. Matsukura, and H. Ohno, Nat. Mater. 9, 721 (2010).

[8] J. M. Shaw, H. T. Nembach, and T. J. Silva, Phys. Rev. B 87, 054416 (2013).

[9] A. Manchon, H. C. Koo, J. Nitta, S. Frolov, and R. A. Duine, Nat. Mater. 14, 871 (2015).

[10] N. Nakajima, T. Koide, T. Shidara, H. Miyauchi, H. Fukutani, A. Fujimori, K. Iio, T. Katayama, M. Nyvlt, and Y. Suzuki, Phys. Rev. Lett. 81, 5229 (1998).

[11] G. H. O. Daalderop, P. J. Kelly, and M. F. H. Schuurmans, Phys. Rev. B 50, 9989 (1994).

[12] P. Bruno, Phys. Rev. B 39, 865 (1989); J. Stöhr, J. Magn. Magn. Mater. 200, 470 (1999).

[13] G. Dresselhaus, Phys. Rev. 100, 580 (1955).

[14] H. Nakamura, T. Koga, and T. Kimura, Phys. Rev. Lett. 108, 206601 (2012).

[15] Y. Tokatly A. Bychkov, and E. I. Rashba, J. Phys. C 17, 6039 (1984).

[16] L. Petersen and P. Hedegård, Surf. Sci. 459, 49 (2000).

[17] S. LaShell, B. A. McDougall, and E. Jensen, Phys. Rev. Lett. 77, 3419 (1996); H. Cercellier, C. Didiot, Y. Fagot-Revurat, B. Kierren, L. Moreau, D. Malterre, and F. Reinert, Phys. Rev. B 73, 195413 (2006).

[18] O. Krupin, G. Bihlmayer, K. Starke, S. Gorovikov, J. E. Prieto, K. Döbrich, S. Blügel, and G. Kaindl, Phys. Rev. B 71, 201403(R) (2005).

[19] C. R. Ast, J. Henk, A. Ernst, L. Moreschini, M. C. Falub, D. Pacilé, P. Bruno, K. Kern, and M. Grioni, Phys. Rev. Lett. 98, 186807 (2007); A. Takayama, T. Sato, S. Souma, and T. Takahashi, ibid. 106, 166401 (2011).

[20] I. Gierz, B. Stadtmüller, J. Vuorinen, M. Lindroos, F. Meier, J. H. Dil, K. Kern, and C. R. Ast, Phys. Rev. B 81, 245430 (2010).
[21] D. Hsieh, D. Qian, L. Wray, Y. Xia, Y. S. Hor, R. J. Cava, and M. Z. Hasan, Nature (London) 452, 970 (2008); Y. L. Chen, J. G. Analytis, J.-H. Chu, Z. K. Liu, S.-K. Mo, X. L. Qi, H. J. Zhang, D. H. Lu, X. Dai, Z. Fang, S. C. Zhang, I. R. Fisher, Z. Hussain, and Z.-X. Shen, Science 325, 178 (2009).

[22] I. E. Dzialoshinskii, Sov. Phys. JETP 5, 1259 (1957); T. Moriya, Phys. Rev. 120, 91 (1960).

[23] M. Bode, E. Y. Vedmedenko, K. von Bergmann, A. Kubetzka, P. Ferriani, S. Heinze, and R. Wiesendanger, Nat. Mater. 5, 477 (2006); M. Bode, M. Heide, K. von Bergmann, P. Ferriani, S. Heinze, G. Bihlmayer, A. Kubetzka, O. Pietzsch, S. Blügel, and R. Wiesendanger, Nature (London) 447, 190 (2007); P. Ferriani, K. von Bergmann, E. Y. Vedmedenko, S. Heinze, M. Bode, M. Heide, G. Bihlmayer, S. Blügel, and R. Wiesendanger, Phys. Rev. Lett. 101, 027201 (2008).

[24] M. Heide, G. Bihlmayer, and S. Blügel, Phys. Rev. B 78, 140403 (2008); F. Schubert, Y. Mokrousov, P. Ferriani, and S. Heinze, ibid. 83, 165442 (2011).

[25] U. K. Rössler, A. N. Bogdanov, and C. Pfleiderer, Nature (London) 442, 797 (2006); N. Nagaosa and Y. Tokura, Nat. Nanotechnol. 8, 899 (2013); S. Heinze, K. von Bergmann, M. Menzel, J. Brede, A. Kubetzka, R. Wiesendanger, G. Bihlmayer, and S. Blügel, Nat. Phys. 7, 713 (2011).

[26] G. Chen, J. Zhu, A. Quesada, J. Li, A. T. N'Diaye, Y. Huo, T. P. Ma, Y. Chen, H. Y. Kwon, C. Won, Z. Q. Qiu, A. K. Schmid, and Y. Z. Wu, Phys. Rev. Lett. 110, 177204 (2013); G. Chen, T. Ma, A. T. N'Diaye, H. Kwon, C. Won, Y. Wu, and A. K. Schmid, Nat. Commun. 4, 2671 (2013).

[27] A. Thiaville, S. Rohart, E. Jué, V. Cros, and A. Fert, Europhys. Lett. 100, 57002 (2012).

[28] K.-S. Ryu, L. Thomas, S. H. Yang, and S. S. P. Parkin, Nat. Nanotech. 8, 527 (2013); S. Emori, U. Bauer, S.-M. Ahn, E. Martinez, and G. S. D. Beach, Nat. Mater. 12, 611 (2013).

[29] V. M. Edelstein, Solid State Commun. 73, 233 (1990).

[30] A. Brataas and K. M. D. Hals, Nat. Nanotechnol. 9, 86 (2014); A. Manchon, Nat. Phys. 10, 340 (2014).

[31] B. A. Bernevig and O. Vafek, Phys. Rev. B 72, 033203 (2005); K. Obata and G. Tatara, ibid. 77, 214429 (2008); A. Manchon and S. Zhang, ibid. 78, 212405 (2008); I. Garate and A. H. MacDonald, ibid. 80, 134403 (2009).

[32] X. Wang and A. Manchon, Phys. Rev. Lett. 108, 117201 (2012); E. van der Bijl and R. A. Duine, Phys. Rev. B 86, 094406 (2012); K.-W. Kim, S. M. Seo, J. Ryu, K.-J. Lee, and H.-W. Lee, ibid. 85, 180404 (2012); D. A. Pesin and A. H. MacDonald, ibid. 86, 014416 (2012); K. M. D. Hals and A. Brataas, ibid. 88, 085423 (2013); C. Ortiz Pauyac, X. Wang, M. Chshiev, and A. Manchon, Appl. Phys. Lett. 102, 252403 (2013); H. Li, X. Wang, F. Dogan, and A. Manchon, ibid. 102, 192411 (2013); P. M. Haney, H.-W. Lee, K.-J. Lee, A. Manchon, and M. D. Stiles, Phys. Rev. B 87, 174411 (2013)

[33] P. M. Haney, H.-W. Lee, K.-J. Lee, A. Manchon, and M. D. Stiles, Phys. Rev. B 88, 214417 (2013); F. Freimuth, S. Blügel, 
and Y. Mokrousov, ibid. 90, 174423 (2014); G. Géranton, F. Freimuth, S. Blügel, and Y. Mokrousov, ibid. 91, 014417 (2015).

[34] I. M. Miron, G. Gaudin, S. Auffret, B. Rodmacq, A. Schuhl, S. Pizzini, J. Vogel, and P. Gambardella, Nat. Mater. 9, 230 (2010); I. M. Miron, T. Moore, H. Szambolics, L. D. Buda-Prejbeanu, S. Auffret, B. Rodmacq, S. Pizzini, J. Vogel, M. Bonfim, A. Schuhl, and G. Gaudin, ibid. 10, 419 (2011); I. M. Miron, K. Garello, G. Gaudin, P.-J. Zermatten, M. V. Costache, S. Auffret, S. Bandiera, B. Rodmacq, A. Schuhl, and P. Gambardella, Nature (London) 476, 189 (2011).

[35] L. Liu, T. Moriyama, D. C. Ralph, and R. A. Buhrman, Phys. Rev. Lett. 106, 036601 (2011); L. Liu, O. J. Lee, T. J. Gudmundsen, D. C. Ralph, and R. A. Buhrman, ibid. 109, 096602 (2012); L. Liu, C.-F. Pai, Y. Li, H. W. Tseng, D. C. Ralph, and R. A. Buhrman, Science 336, 555 (2012).

[36] D. Fang, H. Kurebayashi, J. Wunderlich, K. Výborný, L. P. Zârbo, R. P. Campion, A. Casiraghi, B. L. Gallagher, T. Jungwirth, and A. J. Ferguson, Nat. Nanotech. 6, 413 (2011); M. Jamali, K. Narayanapillai, X. Qiu, L. M. Loong, A. Manchon, and H. Yang, Phys. Rev. Lett. 111, 246602 (2013); J. Kim, J. Sinha, M. Hayashi, M. Yamanouchi, S. Fukami, T. Suzuki, S. Mitani, and H. Ohno, Nat. Mater. 12, 240 (2013); X. Fan, J. Wu, Y. P. Chen, M. J. Jerry, H. W. Zhang, and J. Q. Xiao, Nat. Commun. 4, 1799 (2013); K. Garello, I. M. Miron, C. O. Avci, F. Freimuth, Y. Mokrousov, S. Blügel, S. Auffret, O. Boulle, G. Gaudin, and P. Gambardella, Nat. Nanotech. 8, 587 (2013); H. Kurebayashi, J. Sinova, D. Fang, A. C. Irvine, J. Wunderlich, V. Novak, R. P. Campion, B. L. Gallagher, E. K. Vehstedt, L. P. Zârbo, K. Vyborny, A. J. Ferguson, and T. Jungwirth, ibid. 9, 211 (2014).

[37] A. R. Mellnik, J. S. Lee, A. Richardella, J. L. Grab, P. J. Mintun, M. H. Fischer, A. Vaezi, A. Manchon, E.-A. Kim, N. Samarth, and D. C. Ralph, Nature (London) 511, 449 (2014); Y. Fan, P. Upadhyaya, X. Kou, M. Lang, S. Takei, Z. Wang, J. Tang, L. He, L.-T. Chang, M. Montazeri, G. Yu, W. Jiang, T. Nie, R. N. Schwartz, Y. Tserkovnyak, and K. L. Wang, Nat. Mater. 13, 699 (2014).

[38] K.-W. Kim, H.-W. Lee, K.-J. Lee, and M. D. Stiles, Phys. Rev. Lett. 111, 216601 (2013).

[39] G. Bihlmayer, Yu. M. Koroteev, P. M. Echenique, E. V. Chulkov, and S. Blügel, Surf. Sci. 600, 3888 (2006).

[40] G. Bihlmayer, S. Blügel, and E. V. Chulkov, Phys. Rev. B 75, 195414 (2007).
[41] H. Bentmann, T. Kuzumaki, G. Bihlmayer, S. Blügel, E. V. Chulkov, F. Reinert, and K. Sakamoto, Phys. Rev. B 84, 115426 (2011).

[42] I. V. Tokatly, E. E. Krasovskii, and G. Vignale, Phys. Rev. B 91, 035403 (2015).

[43] S. Grytsyuk and U. Schwingenschlögl, Phys. Rev. B 88, 165414 (2013).

[44] M. Montalti, A. Credi, L. Prodi, and M. T. Gandolfi, Handbook of Photochemistry (CRC Press, Taylor \& Francis Group, Boca Raton, USA, 2006), p. 617.

[45] J. P. Perdew, K. Burke, and M. Ernzerhof, Phys. Rev. Lett. 77, 3865 (1996).

[46] G. Kresse and J. Furthmüller, Comput. Mater. Sci. 6, 15 (1996).

[47] G. Kresse and D. Joubert, Phys. Rev. B 59, 1758 (1999).

[48] Certain commercial products are identified in this paper in order to adequately specify the computational procedures. Such identification is not intended to imply recommendation or endorsement by the National Institute of Standards and Technology, nor is it intended to imply that the materials or equipment identified are necessarily the best available for the purpose.

[49] http://www.flapw.de (unpublished).

[50] O. Šipr, S. Bornemann, H. Ebert, S. Mankovsky, J. Vackář, and J. Minár, Phys. Rev. B 88, 064411 (2013).

[51] A. Lehnert, S. Dennler, P. Błoński, S. Rusponi, M. Etzkorn, G. Moulas, P. Bencok, P. Gambardella, H. Brune, and J. Hafner, Phys. Rev. B 82, 094409 (2010).

[52] S. Bornemann, O. Šipr, S. Mankovsky, S. Polesya, J. B. Staunton, W. Wurth, H. Ebert, and J. Minár, Phys. Rev. B 86, 104436 (2012).

[53] L. Szunyogh, J. Magn. Magn. Mater. 165, 254 (1997).

[54] B. Újfalussy, L. Szunyogh, P. Bruno, and P. Weinberger, Phys. Rev. Lett. 77, 1805 (1996).

[55] J.-H. Park, C. H. Kim, H.-W. Lee, and J. H. Han, Phys. Rev. B 87, 041301 (2013).

[56] H. Ishida, Phys. Rev. B 90, 235422 (2014).

[57] E. E. Krasovskii, Phys. Rev. B 90, 115434 (2014).

[58] S. R. Park, C. H. Kim, J. Yu, J. H. Han, and C. Kim, Phys. Rev. Lett. 107, 156803 (2011).

[59] The muffin tin radii $\rho_{i}$ used in the FLEUR calculations were $\rho_{\mathrm{Co}}=2.2 a_{0}, \quad \rho_{\mathrm{H}}=0.5 a_{0}, \quad \rho_{\mathrm{Ir}}=2.41 a_{0}, \quad \rho_{\mathrm{Pt}}=2.4 a_{0}, \quad \rho_{\mathrm{Au}}=$ $2.42 a_{0}, \rho_{\mathrm{Rh}}=2.36 a_{0}, \rho_{\mathrm{Pd}}=2.32 a_{0}$, and $\rho_{\mathrm{Ag}}=2.4 a_{0}$, where $a_{0} \approx 0.0529 \mathrm{~nm}$ is the Bohr radius. 